\title{
Inhibition of calcineurin by FK506 stimulates germinal vesicle breakdown of mouse oocytes in hypoxanthine-supplemented medium
}

\author{
Li Wang ${ }^{1}$, Yan-Hong Zhen ${ }^{1,2}$ ， Xiao-Ming Liu ${ }^{1,3}$ ， Jing Cao ${ }^{1}$ ， Yan-Ling Wang ${ }^{1}$, Li-Jun Huo ${ }^{\text {Corresp. } 1}$ \\ ${ }^{1}$ Key Laboratory of Agricultural Animal Genetics, Breeding and Reproduction, Education Ministry of China, College of Animal Science and Technology, \\ HuaZhong Agriculture University, Wu Han, Hu Bei Province, People's Republic of China \\ 2 Animal Husbandry and Veterinary, Wuhan Agricultural School, Wuhan, Hu Bei Province, People's Republic of China \\ 3 Reproductive Medicine Center, Second Affiliated Hospital of Wenzhou Medical College, Wen zhou, People's Republic of China.
}

Corresponding Author: Li-Jun Huo

Email address: lijunhuo@yahoo.com

Calcineurin $(\mathrm{CN})$ is a serine/threonine phosphatase which plays important roles in meiosis maturation in invertebrate oocytes, however, the role of $\mathrm{CN}$ in mouse oocytes is relatively unexplored. In this study, we examined the expression, localization and functional roles of $\mathrm{CN}$ in mouse oocytes and granulosa cells. The RT-PCR results showed that $\beta$ isoform of calcineurin $A$ subunit ( $C n A$ ) expressed significantly higher than $\alpha$ and $\gamma$ isoforms, and the expression of $C n A \beta$ mRNA decreased obviously in oocytes occurred germinal vesicle breakdown (GVBD). While only B1 of calcineurin B subunit (Cn B) was detected in oocytes and expressed stably during oocytes maturation. The following fluorescence experiment showed that Cn A was mainly located in nucleus of germinal vesicle (GV) stage oocytes and gruanlosa cells, and subsequently dispersed into the entire cytoplasm after GVBD. The decline of $\mathrm{Cn} A$ in oocytes suggested that it may play an important role in GVBD. To further clarify the role of calcineurin during meiotic maturation, FK506 (a calcineurin inhibitor) was used in the culture medium contained hypoxanthine (HX) which could keep mouse oocytes staying at GV stage. As expected, FK506 could induce a significant elevation of GVBD rate and increase the MPF level of denuded oocytes (DOs). Furthermore, FK506 could also play an induction role of GVBD of oocytes in COCs and follicles, and the process could be counteracted by MAPK kinase inhibitor (U0126). Above all, the results implied that calcineurin might play a crucial role in development of mouse oocytes and MPF and MAPK pathways are involved in this process. 
1 Inhibition of calcineurin by FK506 stimulates germinal vesicle breakdown of mouse 2 oocytes in hypoxanthine-supplemented medium

4 Li Wang', Yan-Hong Zhen ${ }^{1,2}$, Xiao-Ming Liu', ${ }^{1,}$, Jing Cao1, Yan-Ling Wang ${ }^{1}$, Li-Jun Huo ${ }^{*}$ 5

61 Key Laboratory of Agricultural Animal Genetics, Breeding and Reproduction, Education 7 Ministry of China, College of Animal Science and Technology, HuaZhong Agricultural

8 University, Wuhan, Hu Bei Province, People's Republic of China

$9 \quad{ }^{2}$ Animal Husbandry and Veterinary, Wuhan Agricultural School, Wuhan, Hu Bei Province, 10 People's Republic of China

$11{ }^{3}$ Reproductive Medicine Center, Second Affiliated Hospital of Wenzhou Medical College,

12 Wen zhou, People's Republic of China

$13 *$ Correspondence author, Email: lijunhuo@yahoo.com

\section{Abstract}

maturation in invertebrate oocytes, however, the role of $\mathrm{CN}$ in mouse oocytes is relatively unexplored. In this study, we examined the expression, localization and functional roles of $\mathrm{CN}$ in mouse oocytes and granulosa cells. The RT-PCR results showed that $\beta$ isoform of calcineurin A subunit (Cn A) expressed significantly higher than $\alpha$ and $\gamma$ isoforms, and the expression of $\mathrm{Cn}$ A $\beta$ mRNA decreased obviously in oocytes occurred germinal vesicle breakdown (GVBD). 
21 While only B1 of calcineurin B subunit (Cn B) was detected in oocytes and expressed stably

22 during oocytes maturation. The following fluorescence experiment showed that $\mathrm{Cn}$ A was

23 mainly located in nucleus of germinal vesicle (GV) stage oocytes and gruanlosa cells, and

24 subsequently dispersed into the entire cytoplasm after GVBD. The decline of $\mathrm{Cn} A$ in oocytes

25 suggested that it may play an important role in GVBD. To further clarify the role of calcineurin

26 during meiotic maturation, FK506 (a calcineurin inhibitor) was used in the culture medium

27 contained hypoxanthine (HX) which could keep mouse oocytes staying at GV stage. As expected,

28 FK506 could induce a significant elevation of GVBD rate and increase the MPF level of

29 denuded oocytes (DOs). Furthermore, FK506 could also play an induction role of GVBD of

oocytes in COCs and follicles, and the process could be counteracted by MAPK kinase inhibitor

(U0126). Above all, the results implied that calcineurin might play a crucial role in development

of mouse oocytes and MPF and MAPK pathways are involved in this process.

\section{Introduction}

Mammalian oocytes arrest at prophase of meiosis I with low MPF (maturation promoting factor) activity in the ovarian follicle. Meiotic resumption from diplotene arrest is morphologically characterized by germinal vesicle breakdown (GVBD) and is mediated by activation of MPF, which consists of CDK1 and cyclinB. It is well known that the activity of phosphatase oppose to CDK1/cyclin B would affect the net MPF activity (Adhikari \& Liu 2014; Hara et al. 2012). Cultured with protein phosphatase inhibitor okadaic acid could induce mouse oocytes overcome the GVBD inhibition (Rime \& Ozon 1990), which also occurs in mouse oocytes microinjected with okadaic or anti-PP1 antibody (Swain et al. 2003). However, further 
42 study is still needed to identify whether other PP type is involved during prophase I arrest of 43 mouse oocytes.

44 Calcineurin $(\mathrm{CN})$, protein phosphatase $2 \mathrm{~B}$, is a serine/threonine protein phosphatase, which 45 is directly regulated by $\mathrm{Ca}^{2+} /$ calmodulin in the cell signaling process and consists of catalytic 46 subunit A and regulatory subunit B. Calcineurin is involved in a variety of important activities, 47 such as movements, fertility, egg laying, and growth in Caenorhabditis elegans (Bandyopadhyay 48 et al. 2002). Calcineurin is also essential for exiting from meiotic arrest in metaphases I and II in Xenopus and Drosophila oocytes. It releases the Xenopus egg meiotic metaphase II arrest by 50 Fizzy/Cdc20, a key regulator of the anaphase-promoting factor6 and Mos-MAP (mitogen51 activated protein) kinase pathway (Mochida \& Hunt 2007). Moreover, the blockage of calcineurin interrupts the progression of oocyte meiosis at the stage of anaphase I (Takeo et al. 2010). Calcineurin along with PP2A is required for ascidians normal egg activation during fertilization (Levasseur et al. 2013). Since ascidians and vertebrates are chordates, it may be inferred that calcineurin may also play an important role in mammalian oocyte maturation and activation.

The mammalian calcineurin subunit $\mathrm{A}$ has three isoforms $-\alpha, \beta, \gamma$ and subunit $\mathrm{B}$ is known of two isoforms and they may have different functions. Knockdown of PPP3CA (calcineurin A $\alpha$ ) protein expression enhanced vascular endothelial growth factor (VEGF)-stimulated, but not fibroblast growth factor 2 (FGF2)-stimulated and attenuated FGF2-induced MAPK3/1 and AKT1 phosphorylation (Hand et al. 2005). When PPP3CB (calcineurin A $\beta$ ) coexpressed with cAMP-specific 3', 5'-cyclic phosphodiesterase (PED4D), the degradation of PED4D will be 
63

64

delayed (Suk et al. 2013). PPP3CC (calcineurin A $\gamma$ ) gene might be a susceptibility gene for human bipolar affective disorder (Mathieu et al. 2008). PPP3R1 (calcineurin B1) are reported to funciton in alzheimer's disease (Peterson et al. 2011) and muscle volume response (Hand et al. 2005). While PPP3R2 (calcineurin B2) is known specific expressed in testes (Ueki et al. 1992). Calcineurin has been found in many types of mammal somatic cells. However, there is very little information about its expression and distribution in mammal oocytes, especially mouse oocyte. Recently, it is reported that both calcineurin subunit A and B are localized in porcine oocytes during growth and meiotic maturation (Tumova et al. 2013). Two potent specific inhibitors of calcineurin has been found which could influence the meiosis of pig oocytes as well as enhance the maturation of growing pig oocytes with partial meiotic competence and delay the maturation of fully grown pig oocytes with full meiotic competence (Petr et al. 2013). While, other study shows that calcineurin catalytic subunit is undetectable in M II mouse oocytes and calcineurin inhibitors (CSA, FK506 or CN-inhibitory peptide) cannot prevent meiotic exit (Suzuki et al. 2010).This implies that calcineurin plays no part in mouse oocyte activation, but little is known about expression and function of calcineurin catalytic subunit in mouse oocyte before M II stage. Furthermore, interactions between oocyte and its follicular component in vivo have not been considered. Consequently, it cannot be extended to CN's function on mouse oocytes maturation in vivo.

As mouse oocytes could undergo spontaneous maturation in vitro culture, hypoxanthine (HX) is widely used to keep oocytes staying at GV stage in vitro to imitate the in vivo oocytes maturation arrest (Wigglesworth et al. 2013). Calcineurin inhibitors, tacrolimus (FK506) and 
84

85

86

87

cyclosporin A (CSA), are widely used in renal transplantation as immunosuppressive drugs (Vanhove et al. 2016) and delay the destruction of cyclins, the global dephosphorylation of Mphase-specific phosphoproteins and the reformation of a fully functional nuclear envelope in study of calcineurin function in oocytes (Mochida \& Hunt 2007; Nishiyama et al. 2007).. Therefore, the aim of this study is to investigate the role of calcineurin in regulation of mouse oocyte meiosis maturation by using FK506 and detect the expression pattern and distribution of calcineurin during oocyte maturation. We found that calcineurin A expressed in mouse oocyte and granulosa cells and inhibition of calcineurin could induce GVBD of oocytes. The results of this study were helpful to understand the regulatory functions of calcineurin on mouse oocytes and provide important information for the regulatory mechanism of folliculogenesis.

\section{Materials and Methods}

\section{Ethics statement and animal feeding regimens}

The animal experimental procedures were approved by HuaZhong Agricultural University Institu tional Animal Care and Use Committee, Wuhan, China (Approval ID: SCXK (Hubei) 2008_0005). Kunming mice (3-4 weeks old) were obtained from the Centre of Laboratory Animals of Hubei Province (Wuhan, PR China). Mice were housed under controlled temperature $\left(25^{\circ} \mathrm{C}\right)$ and lighting $(12 \mathrm{~h}$ light $/ 12 \mathrm{~h}$ darkness $)$ with food and water ad libitum and sacrificed with cervical dislocation method.

\section{Isolation and culture of DOs, COCs and follicles}


104

105

106

107

108

109

110

111

112

113

114

115

116

117

118

119

120

121

122

123

culture medium was added to be convenient for collecting oocytes by straws. 50 fully grown and immature oocytes arrested at the GV stage were cultured in $500 \mu$ l M199 medium (Life Technologies Corporation, Rockville, MD) with 4 mM HX or not.

In the same way, ovaries were collected from 3-4 weeks KM mice injected with 10 IU PMSG (Sansheng Pharmaceutical Corporation, China) for 44 hours, and were punctured by the needles of $1 \mathrm{ml}$ injector. Then COCs with at least 2 or 3 layers granulosa cells or antral follicles (FOs) with a diameter about 300-400 $\mu \mathrm{m}$ were collected from the medium by straws. 50 COCs or FOs were cultured in $500 \mu 1 \mathrm{M} 199$ medium with $4 \mathrm{mM} \mathrm{HX}$.

\section{RT-PCR analysis}

RNA from oocytes and granulosa cells was isolated using Rneasy Plus Micro Kit(QIAGEN, Germany)and RNAprep pure cell kit (Tiangen, Beijing China), respectively. Total RNA was transcribed to cDNA with first strand cDNA synthesis kit (Therm, USA). And quantitative realtime PCR was carried out using SYBR Green (QuantiFast SYBR Green PCR kit (QIAGEN, Germany)) on a Bio-Rad LC480 real-time PCR system. All primer pairs used for real-time PCR are summarized in Table 1.The amplification efficiencies of primers were obtained by making standard curves and efficiencies of PCR amplification were between $90-105 \%$ and the variation was less than $5 \%$. The relative quantification of mRNA expression for each calcineurin isoform was estimated using formula $2^{-\mathrm{DDCt}}$, according to the comparative $\mathrm{Ct}$ method, normalized to the housekeeping gene $(A c t b)$ in each sample.

Table 1. Sequence of primer pairs for quantitative real-time PCR. 


\begin{tabular}{|c|c|c|c|}
\hline \multirow{6}{*}{ Calcineurin A } & \multirow{2}{*}{ Ppp3ca (isoform $\alpha$ ) } & Sense primer & 5'-CAACACTCGCTACCTCTTC-3' \\
\hline & & Antisense primer & 5'-CCATACAGGCGTCATAAA-3' \\
\hline & \multirow{2}{*}{ Ppp3cb (isoform $\beta$ ) } & Sense primer & 5'-TAGTGGAGTGTTGGCTGG-3' \\
\hline & & Antisense primer & 5'-AGTGGTATGTGCGGTGTT-3' \\
\hline & \multirow{2}{*}{ Ppp3cc (isoform $\gamma$ ) } & Sense primer & 5'-CCTCTTGCTGCCCTCTTA-3' \\
\hline & & Antisense primer & 5'-СTTCTCGCTGCCGTAGTC-3' \\
\hline \multirow{4}{*}{ Calcineurin B } & \multirow{2}{*}{ Ppp3r1 (isoform 1) } & Sense primer & 5'-AGGCGAGTTACCCTTTGG-3' \\
\hline & & Antisense primer & 5'-CTCCGTTGCCGTCTGTG-3' \\
\hline & \multirow{2}{*}{ Ppp3r2 (isoform 2) } & Sense primer & 5'-CTCCCAGTTCAGCGTCAA-3' \\
\hline & & Antisense primer & 5'-ATCGCCATCCTTATCCAG-3' \\
\hline \multirow{2}{*}{$A c t b$} & & Sense primer & 5'- CCCATCTACGAGGGCTAT-3' \\
\hline & & Antisense primer & 5'-TGTCACGCACGATTTCC-3' \\
\hline
\end{tabular}

124

125

126

127

128

129 130

\section{Immunohistochemistry}

Mouse ovaries were collected and fixed in Borne's solution for 12-16 hr at RT. The fixed mouse ovaries were dehydrated in graded ethanol, dealcoholized with xylene, soaked in methyl salicylate for 12-16 hr, and embedded in paraffin. The paraffin-embedded tissues were sectioned into 4- $\mu \mathrm{m}$ thick slices, mounted onto poly-L-lysine-coated slides and dried at $42^{\circ} \mathrm{C}$ for $2-4 \mathrm{hr}$ in air, subsequently transferred into $60^{\circ} \mathrm{C}$ overnight in incubator. The sections were deparaffinized and rehydrated in graded ethanol, soaked in 3\% hydrogen peroxide for $30 \mathrm{~min}$ to remove 
131

endogenous peroxidase and washed in $\mathrm{ddH} 2 \mathrm{O}$ two times for 5 min each followed by PBS two times for 5 min each. The sections were boiled in $0.01 \mathrm{M}$ sodium citrate $(\mathrm{pH}$ 6.0) three times for 2 min each at 5-min intervals for antigen retrieval. Then the samples were blocked by donkey serum for $30 \mathrm{~min}$ at $\mathrm{RT}$, then incubated overnight at $4^{\circ} \mathrm{C}$ with anti-Cacineurin A antibody (Santa Cruz Biotechnology, USA,SC9070) (1:100 dilution) or rabbit IgG (Santa Cruz Biotechnology, USA) (1:100 dilution) as control in blocking solution. The sections washed three times for $5 \mathrm{~min}$ each in $0.1 \%$ Tween-20 in PBS and the samples were incubated with goat anti-rabbit biotin-SPconjugated antibody (Guge Biotechnology Corporation, Wuhan, China) for 30 min at RT. The sections washed three times for $5 \mathrm{~min}$ each in $0.1 \%$ Tween-20 in PBS and the samples were incubated with $\mathrm{SABC}$ for 30 min at $37^{\circ} \mathrm{C}$. After washing 4-5 times for 5 min each in PBS, the immune reactive signals were detected using DAB Map Kit (Guge Biotechnology Corporation, Wuhan, China) for 3-5 min. The sections washed four times for 5 min each in ddH2O. The samples were stained by hematoxylin for $2 \mathrm{~min}$, differentiated by $5 \%$ hydrochloric acid for $8 \mathrm{~s}$, washed by ddH2O three times for $5 \mathrm{~min}$ each and washed by PBS three times for $5 \mathrm{~min}$ each to make the stain of nucleus return to blue. The sections were dehydrated in graded ethanol, dealcoholized with xylene, and sealed with neutral resin and cover glass. The sections were put in $37^{\circ} \mathrm{C}$ for $24 \mathrm{~h}$ to $48 \mathrm{hr}$ and were observed by microscope with Nomarski optics and digital camera.

\section{Immunofluorescence and confocal microscopic techniques}


151 GVBD or M II stages, were collected for immunofluorescent staining. Oocytes were fixed in 4\% 152 paraformaldehyde in PBS (Phosphate Buffered Saline) for $1 \mathrm{~h}$ at RT (room temperature). After 153 washing 3 times in wash buffer (PBS containing 2\% BSA), oocytes were treated with $0.5 \%$ 154 Triton X-100 in PBS for 1-1.5h at RT, blocked in PBS containing 5\% BSA for $1 \mathrm{~h}$ at RT and 155 incubated overnight at $4^{\circ} \mathrm{C}$ with anti-Cacineurin $\mathrm{A}$ antibody (Santa Cruz Biotechnology, 156 USA,SC9070) (1:100 dilution) or rabbit IgG (Santa Cruz Biotechnology, USA) (1:100 dilution) 157 as control in blocking solution. Then oocytes were incubated with a FITC-conjugated goat anti158 rabbit antibody (Boster Co, Wuhan, China) (1:100 dilution) in dark after washing 3times in 15 $159 \mathrm{~min}$, nuclei were stained in washing buffer containing $10 \mu \mathrm{g} / \mathrm{ml}$ PI (Propidium Iodide) for 10 160 min. After immunofluorescent staining, oocytes were mounted on slides in DABCO and 161 fluorescent signals were detected using confocal microscope.

162 Granulosa cells adherent to coverslip were fixed in 4\% paraformaldehyde in PBS for 30min 163 at RT and washed for 3times. After treating with $0.5 \%$ Triton X-100 in PBS for 15-20 min at RT, 164 granulosa cells were blocked in 5\% BSA with PBS for $30 \mathrm{~min}$ at RT and incubated overnight at $1654^{\circ} \mathrm{C}$ with anti-Calcienurin A antibody (Santa Cruz Biotechnology, USA,SC9070) (1:100 dilution) 166 or rabbit IgG (1:100 dilution) as control in blocking solution, then incubated with a FITC167 conjugated goat anti-rabbit antibody (1:100 dilution) in dark and washed 3times in 15 min, later 168 nuclei were stained in washing buffer containing $10 \mu \mathrm{g} / \mathrm{ml}$ PI for 10 min. After 169 immunofluorescent staining, coverslips were mounted on slides with DABCO and fluorescent 170 signals were detected using confocal microscope. 
171

172

173

174

175

176

177

178

179

180

181

182

183

184

185

186

187

188

189

\section{Western blot analysis}

A total of 200 fully grown GV, GVBD and M II oocytes were collected and lysed in 2X SDS sample buffer and boiled in water for $5 \mathrm{~min}$. Total protein was concentrated by $5 \%$ SDSPAGE (SDS-polyacrylamide gel electrophoresis) for $30 \mathrm{~min}$ at $90 \mathrm{~V}$, and then was separated by 12\% SDS-PAGE for $90 \mathrm{~min}$ at $120 \mathrm{~V}$ and electrophoretically transferred to a PVDF membrane for $1 \mathrm{~h}$ at $200 \mathrm{~mA}$. After washing in TBS (Tris-HCl Buffer Saline) for 3 times, the membrane was blocked in TBST (TBS with $0.1 \%$ Tween 20) containing 5\% skim milk for $1-2 \mathrm{~h}$, and then incubated with a rabbit anti-Calcineurin A antibody (Santa Cruz Biotechnology, USA, SC9070) (1:200 dilution) or a mouse anti- $\beta$-actin (Santa Cruz Biotechnology, USA, SC69879) (1:1000 dilution) in TBST overnight at $4^{\circ} \mathrm{C}$. After washing 3 times in TBST, the membrane was incubated with an HRP-conjugated anti-rabbit or mouse secondary antibody (Boster Corporation, Wuhan, China) (1:2000 dilution) in TBST for $1 \mathrm{~h}$ at RT. The membrane was washed 3 times with TBST and processed with the ECL (Enhanced Chemilumidescence) detection system. ACTB was detected as an internal control.

\section{MPF activity assay}

After treating with or without FK506 for $12 \mathrm{~h}, 100$ oocytes were lysed with $30 \mu 1$ of radio immune precipitation assay (RIPA) buffer supplemented with a protease inhibitor cocktail (Sigma). Samples were lysed on ice for 5-10 min, and then centrifuged at $4^{\circ} \mathrm{C}$ at $12,000 \mathrm{rpm}$ for 15 min. The supernatant was collected and stored at $-80^{\circ} \mathrm{C}$ until use. The level of MPF was 
190

191

192

193

194

195

196

197

198

199

200

201

202

203

204

205

206

207

determined using the Mouse MPF ELISA Kit (Kexing Biotechnology Company, China)

following the manufacturer's protocol and each sample was tested with three repeats. All other chemicals were purchased from Sigma Aldrich, unless stated otherwise.

\section{Statistical Analysis}

Each experiment was repeated at least three times and data were presented as mean $\pm \mathrm{SEM}$ (standard error of the mean). Differences between groups were analyzed using one-way ANOVA followed by Tukey's Honest Significant Difference (HSD) test using SPSS (Version 17.0; SPSS, Chicago, IL).P values $<0.05$ were considered to be statistically significant.

\section{Results}

\section{Identification of the mRNA expression level for isoforms of calcineurin subunits}

To identify the different expression pattern ofcalcineurin' subunits during oocytes meiotic maturation, three developmental stages (GV, GVBD and PB1 extrusion) oocytes and granulosa cells were isolated for detection.

Three isoforms ( $\alpha, \beta$ and $\gamma$ ) of calcineurin A subunit mRNA were detected in oocytes and the mRNA level of $\beta$ isoform was significantly higher than $\alpha$ and $\gamma$ during meiotic maturation. While only B1 isoform of calcineurin B was found in oocytes and expressed during oocyte maturation constantly (Fig. 1 A). Similar with oocytes, three isoforms of calcineurin A subunit were detected in granulosa cells and the mRNA level of $\mathrm{Cn} A \beta$ was significantly higher than $\mathrm{Cn}$ $\mathrm{A} \alpha$ and $\mathrm{Cn} A \gamma$. However, mRNAs of two isoforms of calcineurin B (B1 and B2) subunit were 
209

210 (Fig.1 B).

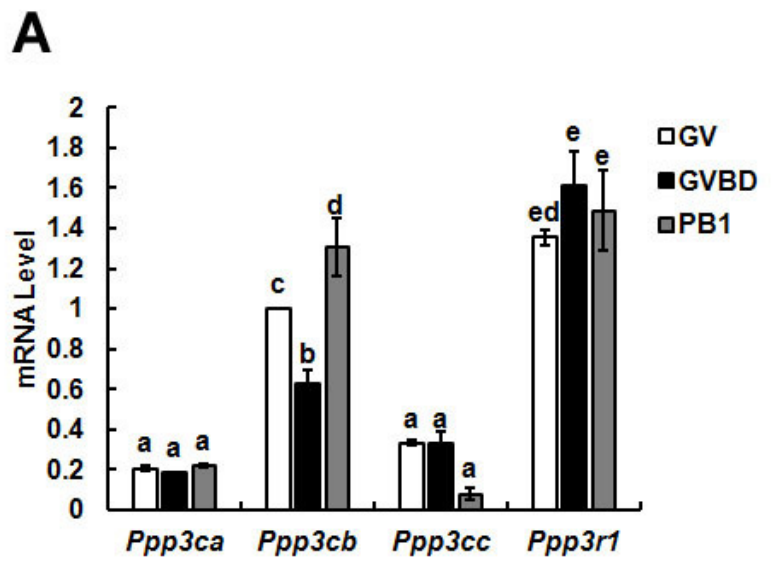

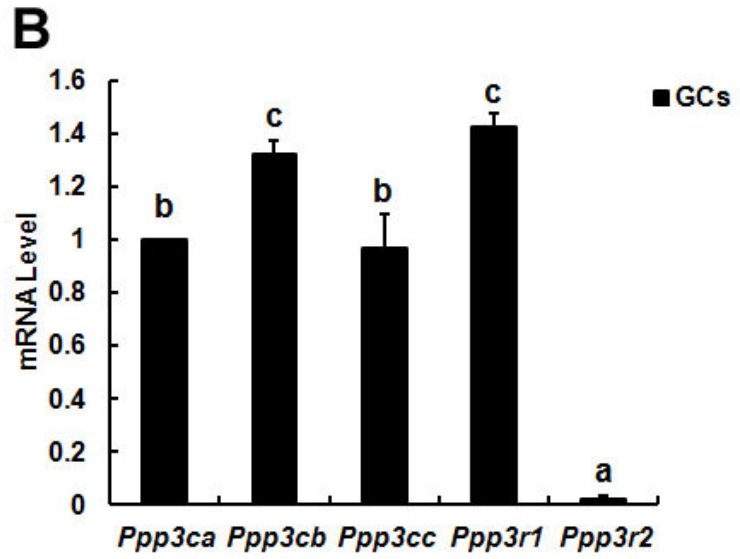

211 relative mRNA levels represent the amount of mRNA expression normalised to Actb. The comparison of calcineurin mRNA expression was made between each oocytes' stage and oocytes in one stage, so was in

\section{Localization and protein expression of calcineurin $A$ in oocytes and granulosa cells}

The above study showed that Cn A $\beta$ mRNA level decreased significantly after GVBD,

219 which suggested $\mathrm{Cn}$ A might play important roles during GVBD. Thus we examined the 220 localization and expression of $\mathrm{Cn} \mathrm{A}$ in mouse ovary. Positive reaction of calcineurin $\mathrm{A}$ was

221 found in different stages of follicles (Fig.2A). Furthermore, immunocytochemical evaluation

222 showed the present of calcineurin A subunit in different stages of oocytes and granulosa cells 
223 and was expressed in nucleus of fully grown GV stage oocytes and granulosa cells, while

224 homogenously dispersed into cytoplasm after GVBD (Fig.2B and E).Western blot analysis

225 showed that the expression of calcineurin A subunit at GV and PB1 extrusion stages of oocytes

226 was significantly higher than GVBD stage oocytes (Fig.2C and D), which was consistent with

227 the mRNA expression of calcineurin A.
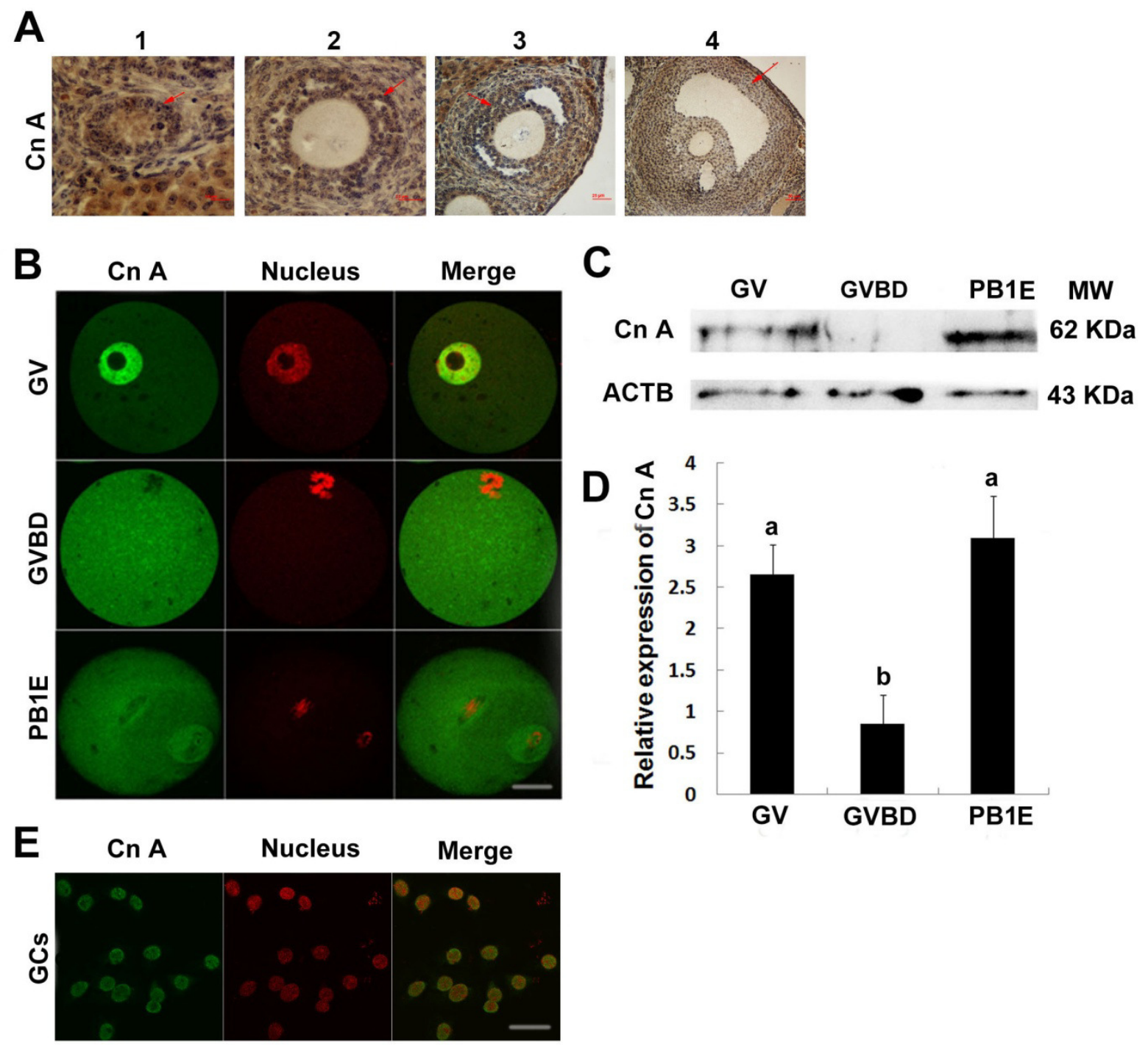

229 Fig. 2 Expression and location of calcineurin A subunit in follicles, oocytes and granulosa cells. A:

230 Calcineurin A subunit was detected by immunohistochemistry in different stage of follicles (1: primordial 
231

232

233

234

235

236

237

238

239

240

241

242

243

244

245

246

247

248

249

250

follicle, 2: primary follicle, 3: small antral follicle, 4: big antral follicle), which were pointed out by red arrows.

B: Cellular localization of calcineurin A subunit was detected by immunofluorescence in GV, GVBD and PB1

extrusion oocytes. Scale bar, $20 \mu \mathrm{m}$. C D: Calcineurin A subunit protein expression was confirmed by Western

blot in GV, GVBD and PB1 extrusion oocytes and the protein ratios were analyzed according to the western

blot results. "a" and "b" represents $P<0.05$. E: Cellular localization of calcineurin A subunit was detected by

immunofluorescence in granulosa cells. Scale bar, $20 \mu \mathrm{m}$.

\section{Inhibition of calcineurin by FK506 could induce the GVBD of oocytes}

To further study the functional role of calcineurin in the development of oocyte,DOs (denuded oocytes) were incubated in basic medium (without HX) containing different concentration of FK506. Since 50 $\mu$ M FK506 could lead to abnormal cell morphology of oocytes (data not showed), we used $5 \mu \mathrm{M}$ and $25 \mu \mathrm{M}$ FK506 to culture DOs and examined the GVBD rate after 2 hours. The results showed that $5 \mu \mathrm{M}$ and $25 \mu \mathrm{M}$ could not disturb the GVBD of oocytes (Fig.3A). While, when DOs were cultured in medium containing 4mM HX for 12 hours, $25 \mu \mathrm{M}$ FK506 could significantly increase the GVBD\% $(54.91 \pm 1.47 \%)$ compared to the control group(37.93 $\pm 2.31 \%)$, as well as $5 \mu \mathrm{M}$ FK506 (45.81 $\pm 1.59 \%)($ Fig.3B).

As calcineurin was expressed both in the oocytes and granulosa cells, we supposed that calcineurin may participate in the communication between oocyte and cumulus cells. To confirm the hypothesis COCs (cumulus oocyte complexes) were cultured in medium with HX and FK506, and GVBD rates were examined after 22 hours. COCs cultured with FK506 and HX showed an significantly increase of GVBD $\%(83.15 \pm 3.28 \%)$ compared to control group $(58.13 \pm 6.74 \%)$, 
251 and showed no significant difference with FSH group (88.85 $\pm 6.16 \%$ ) (Fig.3C). However,

252 inhibition of calcineurin by FK506 had no effect on cumulus expansion (Fig.3D). These results

253 suggested that calcineurin might play an important role in maintaining the oocytes stayed at GV

254 stage. Antral follicles, which had a diameter about 300-400 $\mu \mathrm{m}$ and contained about the same

255 condition of COCs as we cultured, were cultured by $25 \mu \mathrm{M}$ FK506 to confirm that $25 \mu \mathrm{M}$ FK506

256 also could induce the GVBD of oocytes from the antral follicles after culture for 22h (Fig.3E).

A

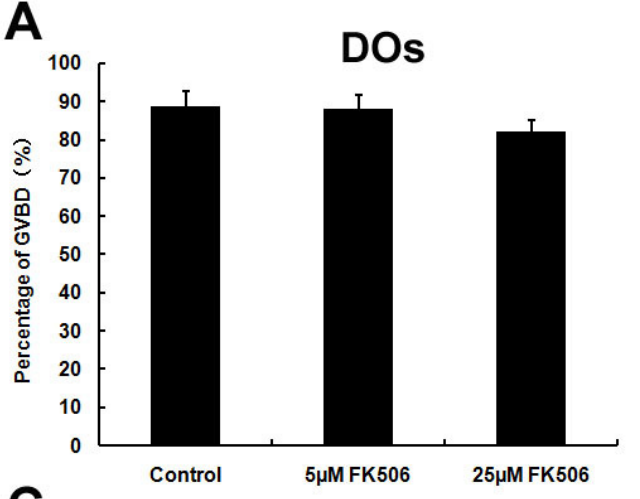

C

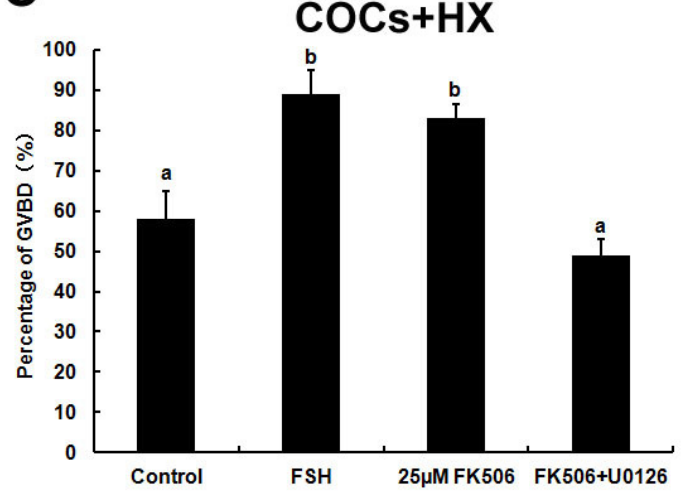

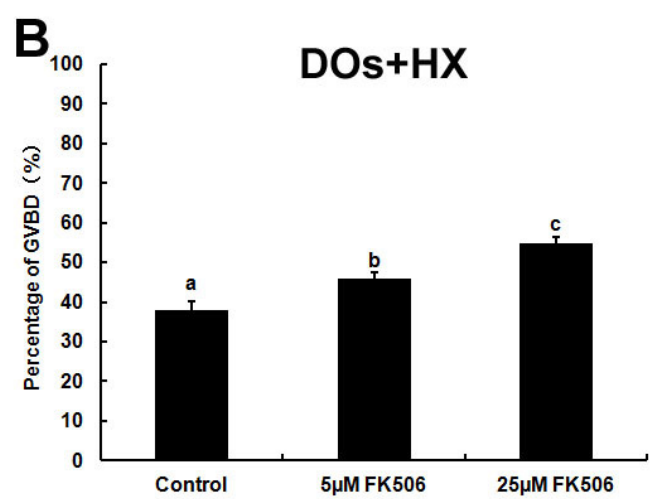

E

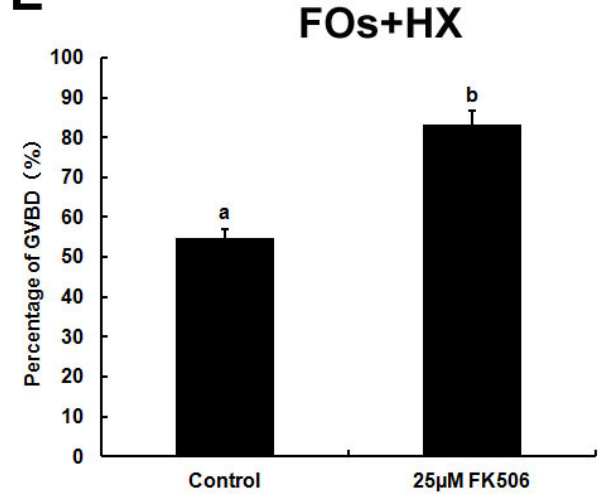

\section{D}

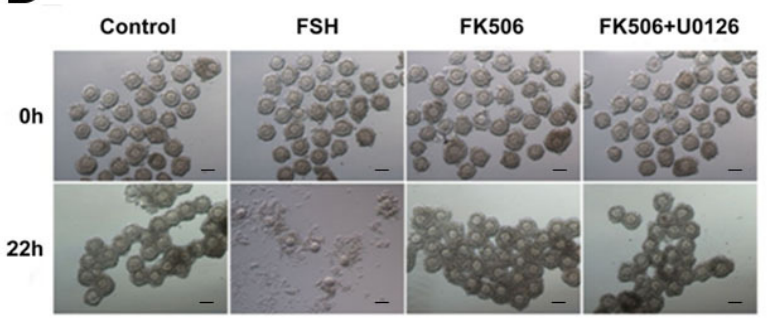

Fig. 3 The effects of FK506 on GVBD rate of oocytes and cumulus expansion. A B: The percentage of GVBD 
259 in oocytes cultured with different concentration of FK506 in medium with or without 4 mM HX. C D: The

260 GVBD percentage of oocytes from COCs cultured with FSH, $25 \mu \mathrm{M}$ FK506 or U0126 $+25 \mu \mathrm{M}$ FK506 in

261 medium with $4 \mathrm{mM}$ HX and the cumulus expansion was examined. Scale bar, $100 \mu \mathrm{m}$. E: The GVBD

262 percentage of oocytes from FOs cultured with $25 \mu \mathrm{M}$ FK506 in medium contain $4 \mathrm{mM}$ HX. Data presented as

263 mean \pm standard error of the mean, from three independent experiments. "a" and "b" , "a" and "b" or "b" and

264 “c" represents $\mathrm{P}<0.05$.

265 FK506 increased MPF level in oocyte which could be reduced by U0126

266 It has been well established that MPF and MPAK are important pathways in meiotic

267 maturation. To further test whether there was feedback regulation between MPF and calcineurin

268 in oocytes, MPF concentration was detected by ELISA. The results showed that the MPF

269 concentration increased significantly after $25 \mu \mathrm{M}$ FK506 treating for 12 hours and partially

270 decreased by MAPK inhibitor U0126(Fig. 4). These results suggest that both MAPK and MPF

271 are associated with the regulation of calcineurin in oocytes. 
272

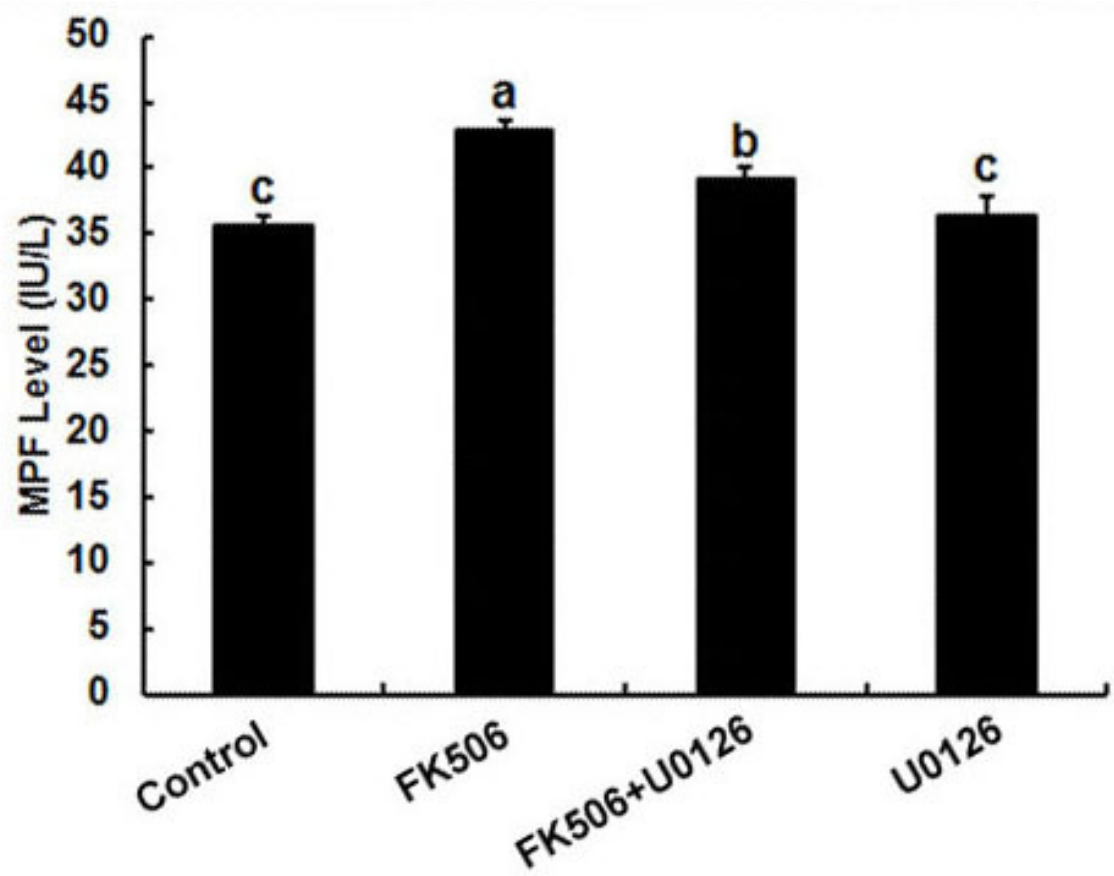

Fig. 4 Inhibition of calcineurin by FK506 increased the concentration of MPF in oocytes. Concentration of MPF was detected after culturing in $25 \mu \mathrm{M}$ FK506 for $12 \mathrm{~h}$. Data presented as mean \pm SEM, from three independent experiments. "a" and "b" , "a" and "b" or "b" and "c" represents $\mathrm{P}<0.05$.

\section{Discussion}

Previous studies have demonstrated that calcineurin is required for Xenopus egg meiotic metaphase II resumption and is necessary for the completion of oocyte meiosis in Drosophila (Mochida \& Hunt 2007; Takeo et al. 2010). But little information exists about the role of calcineurin in mammal oocytes maturation. Therefore, we detected the expression, location and function of calcineurin in mouse ovary. The results showed that calcineurin was expressed in oocytes and granulosa cells of all stages of follicles and inhibition of calcineurin could induce mouse oocytes GVBD, which indicated that calcineurin is an important regulator in mouse ovary. 
$\gamma$ isoform, while, only isoform $\beta$ and $\gamma$ were detected in pig oocytes and cumulus cells (Tumova

et al. 2013).Furthermore, the expression of isoform $\beta$ of calcineurin subunit $A$ decreased significantly after GVBD of mouse oocytes, which was consistent with western blotting analysis that the protein expression of calcineurin A subunit at GV stage oocytes was significantly higher than GVBD stage oocytes. Besides, the isoform 1, but not isoform 2, of calcineurin subunit B was detected in mouse oocytes, as opposed to porcine oocytes where testis-specific calcineurin isoform 2 was detected (Tumova et al. 2013). Cn B mRNA was expressed constantly during oocytes maturation. These results suggested that calcineurin might play an important role in

GVBD of mouse oocytes, especially the isoform $\beta$ of calcineurin subunit A. maturation and started to homogenise during the germinal vesicle breakdown (Tumova et al. 2013). This localization might due to its special function on cortical granule excocytosis during pig oocyte activation (Tůmová et al. 2016). However, the present results showed $\mathrm{Cn}$ A was mainly localized in germinal vesicle of GV stage mouse oocyte and homogenously dispersed into cytoplasm after GVBD. It suggested that calcineurin might function directly in nucleus as an transcriptional regulation factor. Moreover, it also occurred in the nucleus of granulosa cells. We 
306

307

308

309

310

311

312

313

314

315

316

317

318

319

320

321

322

323

324

325

326

cells and oocyte communication. As expected, the GVBD rate of oocytes from COCs in FK506 group increased significantly compared to control group, so was in oocytes from follicles. But FK506 had no significant effect on inducing the expansion of COCs. It is reported that PDE4D is expressed in granulosa cells but not in oocytes and inhibition of PDE4D induces oocyte maturation by increasing cAMP levels in granulosa cells (Tsafriri et al. 1996). Recent study showed that calcineurin could bind directly to and inhibit the proteosomal degradation of PDE4D, and the levels of PDE4D expression were potentiated when it coexpressed with CnA $\beta$ (Zhu et al. 2010). Thence, these findings indicated that inhibition of calcineurin by FK506 might induce oocyte maturation through promoting the degradation of PDE4D in cumulus cells.

It has been well established that MPF and the mitogen activated protein kinase (MAPK) are important pathways in meiotic maturation. Though MPF and MAPK activities are dispensable for breakdown of the oocyte GV membrane (Endo et al. 2006), it is not required for the activation of MPF during the first meiosis (Sugiura et al. 2006), when injected into GV stage mouse oocytes, the changes in activity of a cytoplasmic MPF are capable of inducing GVBD and PB1 extrusion (Nakano \& Kubo 2000). Conditional knockout ERK1/2 in granulosa cells in vivo resulted in LH-induced oocyte resumption of meiosis, ovulation, and luteinization failure (Fan et al. 2009). The activation of MAPK by FSH in cumulus cells can induce GVBD of mouse oocytes (Fan et al. 2004). Protein phosphatases can play an important role in mechanisms of MPF activation and inactivation. Our results showed that FK506 increased GVBD rate and MPF level in mouse DOs which could be reduced by U0126, the specific inhibitor of MAPK. This was consistent with the report that MAPK could override phosphodiesterase inhibitor 3-isobutyl-1- 
327 methylxanthine (IBMX) induced mouse oocytes maturation arrest (Choi et al. 1996). The

328 functions and relationship between MAPK and MPF are complicate in oocyte development.

329 MAPK and MPF play crucial roles in spindle assembly checkpoint, increased activity of MAPK

330 could cause disturbance of microtubules and irregularly pull chromosomes disperse over the

331 spindle and activate spindle assembly checkpoint. Interestingly, during spontaneous activation,

332 MAPK first decline and then increase and the activity of MPF fluctuates similarly but always

333 changes ahead of the MAPK activity (Cui et al. 2012). In addition, activation of MAPK and

334 MPF by c-erbB and c-myb induced oocyte GVBD (Zheng et al. 2012; Zheng et al. 2008).

335 Inhibition of MAPK by U0126 could decrease the GVBD rate and MPF expression induced by

336 FK506. FK506 could induce a rapid and transient increase of ERK1/2 phosphorylationin rat

337 renal mesangial cells (Akool el et al. 2012), we can speculate that activation of MAPK and MPF

338 pathways might involve in the FK506 induced oocyte maturation.

339 Thus, we speculated that inhibition of calcineurin by FK506 might induce mouse oocyte

340 GVBD through activating MPF and MAPK pathways and inhibiting PDE4D pathway.

341 Calcineurin may serve as a key regulatory factor in the regulation of development of mouse

342 oocytes in vivo. However, further studies will be needed to validate the biological significance of

343 calcineurin in oocytes development.

\section{References}

Adhikari D, and Liu K. 2014. The regulation of maturation promoting factor during prophase I arrest and meiotic entry in mammalian oocytes. Mol Cell Endocrinol 382:480-487. 10.1016/j.mce.2013.07.027

Akool el S, Gauer S, Osman B, Doller A, Schulz S, Geiger H, Pfeilschifter J, and Eberhardt W. 2012. Cyclosporin A and 
tacrolimus induce renal Erk1/2 pathway via ROS-induced and metalloproteinase-dependent EGF-receptor signaling. Biochem Pharmacol 83:286-295. 10.1016/j.bcp.2011.11.001

Bandyopadhyay J, Lee J, Lee J, Lee JI, Yu JR, Jee C, Cho JH, Jung S, Lee MH, Zannoni S, Singson A, Kim DH, Koo HS, and Ahnn J. 2002. Calcineurin, a calcium/calmodulin-dependent protein phosphatase, is involved in movement, fertility, egg laying, and growth in Caenorhabditis elegans. Mol Biol Cell 13:3281-3293. 10.1091/mbc.E02-01-0005

Choi T, Rulong S, Resau J, Fukasawa K, Matten W, Kuriyama R, Mansour S, Ahn N, and Vande Woude GF. 1996. Mos/mitogen-activated protein kinase can induce early meiotic phenotypes in the absence of maturationpromoting factor: a novel system for analyzing spindle formation during meiosis I. Proc Natl Acad Sci U S A 93:4730-4735.

Cui W, Zhang J, Lian H-Y, Wang H-L, Miao D-Q, Zhang C-X, Luo M-J, and Tan J-H. 2012. Roles of MAPK and spindle assembly checkpoint in spontaneous activation and MIII arrest of rat oocytes. PloS one 7:e32044.

Endo T, Naito K, Kume S, Nishimura Y, Kashima K, and Tojo H. 2006. Activities of maturation-promoting factor (MPF) and mitogen-activated protein kinase (MAPK) are not required for the global histone deacetylation observed after germinal vesicle breakdown (GVBD) in porcine oocytes. Reproduction 131:439-447. 10.1530/rep.1.00924

Fan HY, Huo L, Chen DY, Schatten H, and Sun QY. 2004. Protein kinase C and mitogen-activated protein kinase cascade in mouse cumulus cells: cross talk and effect on meiotic resumption of oocyte. Biol Reprod 70:1178-1187. 10.1095/biolreprod.103.024737

Fan HY, Liu Z, Shimada M, Sterneck E, Johnson PF, Hedrick SM, and Richards JS. 2009. MAPK3/1 (ERK1/2) in ovarian granulosa cells are essential for female fertility. Science 324:938-941. 10.1126/science.1171396

Hand BD, Ferrell RE, Kostek MC, Delmonico MJ, Doldo NA, Bailey JA, Roth SM, and Hurley BF. 2005. Protein Phosphatase 3 (PPP3R1) Gene Polymorphism Influences Muscle Volume Response To Strength Training: 872 Board\# 94 10: 30 AM-12: 00 PM. Medicine \& Science in Sports \& Exercise 37:S164.

Hara M, Abe Y, Tanaka T, Yamamoto T, Okumura E, and Kishimoto T. 2012. Greatwall kinase and cyclin B-Cdk1 are both critical constituents of M-phase-promoting factor. Nat Commun 3:1059. 10.1038/ncomms2062

Levasseur M, Dumollard R, Chambon JP, Hebras C, Sinclair M, Whitaker M, and McDougall A. 2013. Release from meiotic arrest in ascidian eggs requires the activity of two phosphatases but not CaMKII. Development 140:4583-4593. 10.1242/dev.096578

Mathieu F, Miot S, Etain B, El Khoury M-A, Chevalier F, Bellivier F, Leboyer M, Giros B, and Tzavara ET. 2008. Association between the PPP3CC gene, coding for the calcineurin gamma catalytic subunit, and bipolar disorder. Behavioral and Brain Functions 4:1.

Mochida S, and Hunt T. 2007. Calcineurin is required to release Xenopus egg extracts from meiotic M phase. Nature 449:336-340. 10.1038/nature06121

Nakano H, and Kubo H. 2000. Study of the in vitro maturation of mouse oocytes induced by microinjection of maturation promoting factor (MPF). J Assist Reprod Genet 17:67-73.

Nishiyama T, Yoshizaki N, Kishimoto T, and Ohsumi K. 2007. Transient activation of calcineurin is essential to initiate embryonic development in Xenopus laevis. Nature 449:341-345. 10.1038/nature06136

Peterson D, Kauwe J, Crowley J, Corcoran C, Cruchaga C, Goate A, Norton M, Green R, Munger R, and Breitner J. 2011. Support for Genetic Association Between Variants in PPP3R1 and MAPT and Rate of Progression of Alzheimer's Disease. Alzheimer's \& Dementia 7:S192. 
390

391

392

393

394

395

396

397

398

399

400

401

402

403

404

405

406

407

408

409

410

411

412

413

414

415

416

417

418

419

420

421

422

423

424

425

426

427

428

429

430

Petr J, Chmelikova E, Zalmanova T, Tumova L, Kheilova K, Kucerova-Chrpova V, and Jilek F. 2013. Pyrethroids cypermethrin, deltamethrin and fenvalerate have different effects on in vitro maturation of pig oocytes at different stages of growth. Animal 7:134-142. 10.1017/s1751731112001140

Rime $\mathrm{H}$, and Ozon R. 1990. Protein phosphatases are involved in the in vivo activation of histone $\mathrm{H} 1$ kinase in mouse oocyte. Dev Biol 141:115-122.

Sugiura K, Naito K, Endo T, and Tojo H. 2006. Study of germinal vesicle requirement for the normal kinetics of maturation/M-phase-promoting factor activity during porcine oocyte maturation. Biol Reprod 74:593-600. 10.1095/biolreprod.105.046375

Suk HY, Zhou C, Yang TT, Zhu H, Raymond Y, Olabisi O, Yang X, Brancho D, Kim J-Y, and Scherer PE. 2013. Ablation of calcineurin $A \beta$ reveals hyperlipidemia and signaling cross-talks with phosphodiesterases. Journal of Biological Chemistry 288:3477-3488.

Suzuki T, Suzuki E, Yoshida N, Kubo A, Li H, Okuda E, Amanai M, and Perry AC. 2010. Mouse Emi2 as a distinctive regulatory hub in second meiotic metaphase. Development 137:3281-3291. 10.1242/dev.052480

Swain JE, Wang X, Saunders TL, Dunn R, and Smith GD. 2003. Specific inhibition of mouse oocyte nuclear protein phosphatase-1 stimulates germinal vesicle breakdown. Mol Reprod Dev 65:96-103. 10.1002/mrd.10258

Takeo S, Hawley RS, and Aigaki T. 2010. Calcineurin and its regulation by Sra/RCAN is required for completion of meiosis in Drosophila. Dev Biol 344:957-967. 10.1016/j.ydbio.2010.06.011

Tsafriri A, Chun SY, Zhang R, Hsueh AJ, and Conti M. 1996. Oocyte maturation involves compartmentalization and opposing changes of cAMP levels in follicular somatic and germ cells: studies using selective phosphodiesterase inhibitors. Dev Biol 178:393-402. 10.1006/dbio.1996.0226

Tůmová L, Chmelíková E, Žalmanová T, Kučerová-Chrpová V, Romar R, Dvořáková M, Hošková K, and Petr J. 2016. Calcineurin role in porcine oocyte activation. Animal: an international journal of animal bioscience:1-10.

Tumova L, Petr J, Zalmanova T, Chmelikova E, Kott T, Tichovska H, Kucerova-Chrpova V, Hoskova K, and Jilek F. 2013. Calcineurin expression and localisation during porcine oocyte growth and meiotic maturation. Anim Reprod Sci 141:154-163. 10.1016/j.anireprosci.2013.07.011

Ueki K, Muramatsu T, and Kincaid RL. 1992. Structure and expression of two isoforms of the murine calmodulindependent protein phosphatase regulatory subunit (calcineurin B). Biochemical and Biophysical Research Communications 187:537-543.

Vanhove T, Annaert P, and Kuypers DR. 2016. Clinical determinants of calcineurin inhibitor disposition: a mechanistic review. Drug Metab Rev:1-25. 10.3109/03602532.2016.1151037

Wigglesworth K, Lee KB, O'Brien MJ, Peng J, Matzuk MM, and Eppig JJ. 2013. Bidirectional communication between oocytes and ovarian follicular somatic cells is required for meiotic arrest of mammalian oocytes. Proc Natl Acad Sci U S A 110:E3723-3729. 10.1073/pnas.1314829110

Zheng LP, Huang J, Zhang DL, Xu LQ, Li F, Wu L, Liu ZY, and Zheng YH. 2012. c-erbB2 and c-myb induce mouse oocyte maturation involving activation of maturation promoting factor. DNA Cell Biol 31:164-170. 10.1089/dna.2011.1219

Zheng YH, Zheng LP, Li F, Wu L, and Dai YC. 2008. [c-erbB(2) and c-myb induce oocyte maturation via activation of mitogen-activated protein kinase and maturation promoting factor]. Sheng Li Xue Bao 60:97-104.

Zhu H, Suk HY, Yu RY, Brancho D, Olabisi O, Yang TT, Yang X, Zhang J, Moussaif M, Durand JL, Jelicks LA, Kim JY, Scherer PE, Frank PG, Lisanti MP, Calvert JW, Duranski MR, Lefer DJ, Huston E, Baillie GS, Houslay MD, Molkentin JD, Jin J, and Chow CW. 2010. Evolutionarily conserved role of calcineurin in phosphodegron- 
